\title{
Manganese Perovskite Nanoparticles and the Downturn of Inverse Susceptibility above the Curie Temperature
}

\author{
M. Maryško, E. Pollert, O. Kaman, P. Veverka and Z. Jirák \\ Institute of Physics, Academy of Sciences of the Czech Republic, Na Slovance 2,18221 Praha 8, Czech Republic \\ In the $\mathrm{La}_{0.75} \mathrm{Sr}_{0.25} \mathrm{MnO}_{3}$ nanoparticle system for hyperthermia a downturn in the inverse susceptibility above \\ the Curie temperature $T_{\mathrm{c}}$ was observed and interpreted in terms of a finite width of the $T_{\mathrm{c}}$ distribution.
}

PACS numbers: 75.75.Fk

\section{General information}

The magnetic study of the nanoparticles usually comprises the determination of the ZFC and FC susceptibilities which corresponds to the measurement after zero-field and field-cooled procedures, respectively. In the present work however we focus our attention on the region above $T_{\mathrm{c}}$, where in many bulk manganites a downturn of the inverse susceptibility was found and explained by the presence of a Griffith phase [1]. In studying the nanoparticles for hyperthermia we observed the same effect, for which the interpretation was suggested using the distribution of $T_{\mathrm{c}}$.

\section{Results and discussion}

The SQUID magnetometer measurements were performed on the manganite $\mathrm{La}_{0.75} \mathrm{Sr}_{0.25} \mathrm{MnO}_{3}$ in the form of the bulk sample (LSMOB) and two $20 \mathrm{~nm}$ nanoparticle samples LSMO and LSMO@ $@ \mathrm{SiO}_{2}$, where the second one was encapsulated in $\mathrm{SiO}_{2}$ [2]. The temperature dependence of the magnetization $M$ under the applied field $7.95 \mathrm{kA} / \mathrm{m}$ was measured when cooling the sample from 400 to $300 \mathrm{~K}$. Well above $T_{\mathrm{c}}, M$ can be separated to a Curie-Weiss $(\mathrm{C}-\mathrm{W})$ part and a small contribution $M_{\mathrm{imp}}$ arising from ferromagnetic impurities. The FC susceptibility $\chi_{g}$ per unit of the weight and the main experimental result — the quantity $\mathrm{d}\left(1 / \chi_{g}\right) / \mathrm{d} T$ (Fig. 1a) was then evaluated from the corrected values $M-M_{\text {imp }}$. In order to explain the observed downturn of $1 / \chi_{g}$, which manifests itself in a maximum of $\mathrm{d}\left(1 / \chi_{g}\right) / \mathrm{d} T$ we assume a Gauss distribution function $f\left(T_{\mathrm{c}}\right)$ characterized by the average value $T_{\text {ca }}=335 \mathrm{~K}$ and a given dispersion $\sigma$, which is approximately equal to the halfwidth of $f\left(T_{\mathrm{c}}\right)$. This assumption is realistic if we realize that there is a random distribution of the nanoparticle sizes and that $T_{\mathrm{c}}$ depends on the nanoparticle diameter [3]. For a given $T, T_{\mathrm{c}}, g$-factor and spin $S$ the reduced magnetization $M_{\mathrm{r}}=M / M_{0}\left(M_{0}\right.$ is the saturated value of $M$ for $T$ approaching zero) and susceptibility $\chi_{\mathrm{r}}=\chi / M_{0}$

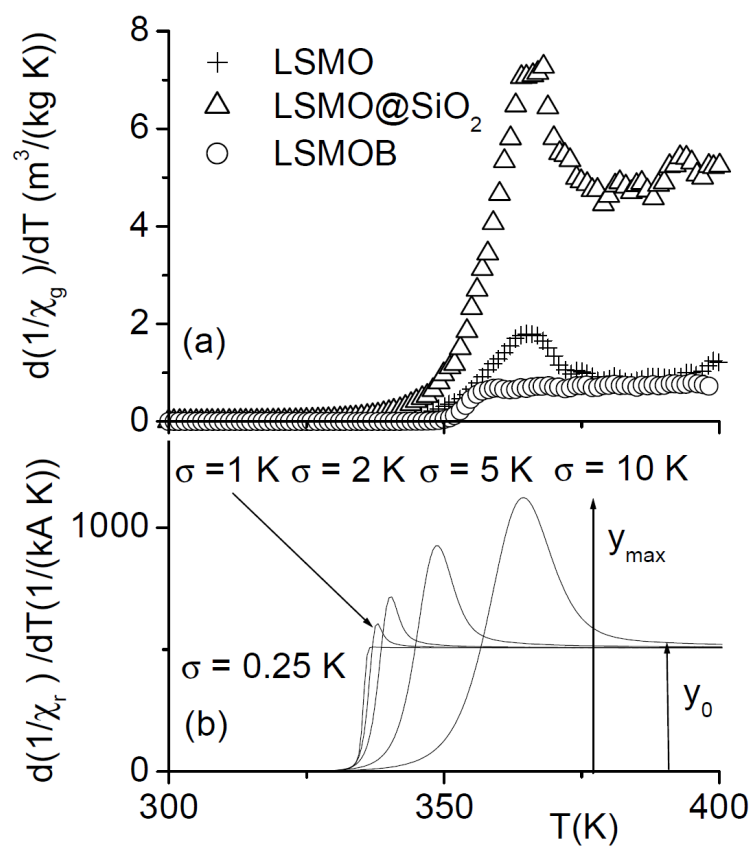

Fig. 1. The derivative $\mathrm{d}\left(1 / \chi_{g}\right) / \mathrm{d} T$ from experiment (a). The calculated values of $\mathrm{d}\left(1 / \chi_{\mathrm{r}}\right) / \mathrm{d} T$ for $H=7.95 \mathrm{kA} / \mathrm{m}, T_{\mathrm{ca}}=335 \mathrm{~K}$ and different values of the dispersion $\sigma(\mathrm{b})$.

can be calculated using the molecular field method. This procedure consists in solving the transcendental equation

$$
M_{\mathrm{r}}=B_{S}\left[S g \mu_{\mathrm{B}}\left(H+\lambda M_{\mathrm{r}}\right) / k_{\mathrm{B}} T\right],
$$

where $\lambda=3 k_{\mathrm{B}} T_{\mathrm{c}} /\left(g \mu_{\mathrm{B}}(S+1)\right)$ and $B_{S}(x)$ is the Brillouin function for spin $S$. Taking $g=2, S=5 / 2$ (this value approximately corresponds to the effective spin deduced from the experiments on the bulk samples) and $\sigma$ as a parameter we determined $M_{\mathrm{r}}$ and finally $\mathrm{d}\left(1 / \chi_{\mathrm{r}}\right) / \mathrm{d} T$ by integrating the product $M_{\mathrm{r}}\left(T, T_{\mathrm{c}}\right)$ over $T_{\mathrm{c}}$ (Fig. 1b). Letting aside absolute values we shall compare the form of the curves displayed in Fig. $1 \mathrm{a}$ and b. For $\sigma=0.25 \mathrm{~K}$ the 
calculated derivative approaches a step function $(\mathrm{C}-\mathrm{W}$ behaviour) and for increasing $\sigma$ the ratio $r=y_{\max } / y_{0}$ increases. For the bulk sample $r \rightarrow 1$ corresponding to $\sigma \rightarrow 0$. For LSMO and LSMO@ $\mathrm{SiO}_{2} r=2.1$ and 1.6 respectively, which suggests a narrower size distribution in the case of the sample containing the encapsulated nanoparticles.

\section{Conclusion}

The downturn of the inverse susceptibility above $T_{\mathrm{c}}$ may give an additional information about the Curie temperature and thus nanoparticle size distribution.

\section{Acknowledgments}

This work is supported by the project ASCR KAN20020061.

\section{References}

[1] M.B. Salamon, S.H. Chun, Phys. Rev. B 68, 014411 (2003).

[2] O. Kaman, E. Pollert, P. Veverka, M. Veverka, E. Hadová, K. Knížek, M. Maryško, P. Kašpar, M. Klementová, V. Grünwaldová, S. Vasseur, R. Epherre, S. Mornet, G. Goglio, E. Duguet, Nanotechnology 20, 275610 (2009).

[3] S. Vasseur, E. Duguet, J. Portier, G. Goglio, S. Mornet, E. Hadová, K. Knížek, M. Maryško, P. Veverka, E. Pollert, J. Magn. Magn. Mater. 302, 315 (2006). 